\title{
Health Related Quality of Life Outcome after Operative Treatment of Traumatic Vertebral Fractures in Geriatric Patients
}

Müller Franz*, Füchtmeier Bernd, Kinner Bernd, Hierl Julia, Nerlich Michael and Neumann Carsten

Department of Trauma and Orthopaedic Surgery, University Medical Center, Regensburg, Germany

\begin{abstract}
Study background: Quality of life survey conducted after the surgical stabilisation of traumatic thoracic and lumbar vertebral fractures in geriatric patients.

Methods: Over a period of 5 years, 42 patients at an age of more than 65 years underwent surgical stabilisation due to an unstable traumatic fracture of the thoracic or lumbar spine. Initially, retrospective empiric data collection was conducted, followed by a prospective radiological and clinical follow-up, including VAS and SF-12.

Results: It was possible to conduct a postoperative radiological and clinical follow-up in 31 out of 42 patients $(74 \%)$ after a mean period of 27.3 months. In 11 out of 42 patients $(26 \%)$, clinical outcome data were collected by relatives. The mean age at the time of surgery was 71.4 years (range 65 to 87 years), and the injury had almost exclusively been stabilised by using a dorsal internal fixator. The surgical revision rate was $12 \%$ ( 5 out of 42 patients), and postoperative 1-year mortality was also $12 \%$ (5 out of 42 patients). At the time of the follow-up, a significant loss of correction was observed in lateral $\mathrm{x}$-ray images when compared to immediate postsurgical results. Quality of life (as measured by SF-12) was significantly reduced at the time of the follow-up compared to a healthy reference population. A significant postoperative decline was also observed with regard to homecare nursing and level of care categories. On the other hand, none of the patients lived in a nursing home, and 35 out of 42 patients $(83 \%)$ were satisfied or very satisfied with the surgical treatment (as measured by a summary measure)

Conclusions: The surgical treatment of traumatic vertebral fractures in geriatric patients resulted in high subjective patient satisfaction post surgery. However, quality of life (SF-12) after an average period of 27.3 months post surgery was still significantly reduced when compared to a healthy reference population.
\end{abstract}

Keywords: Spine; Vertebral fracture; Surgery; Geriatric; Trauma; Outcome

\section{Introduction}

The aging of the population and many risk factors (e.g. low physical activity, low intake of calcium and vitamin D) are especially associated with osteoporotic vertebral fractures in geriatric patients $[1,2]$. However, it is necessary to differentiate between osteoporotic vertebral fractures without trauma and acute injuries with preceding trauma: According to Magerl et al. [3] and their fracture classification, the first ones are mainly type A1 injuries-i.e. generally stable fractures, mostly in the transition area of the thoracic and lumbar spine-that should principally be treated conservatively, even when percutaneous vertebroplasty or kyphoplasty offer minimal invasive surgical options with subjectively good results [4,5]. In contrast, accidents or even minor traumas in geriatric patients will result in severe type A3 (incomplete or complete burst fractures), or even type B or C unstable fractures that require surgical stabilisation. Depending on the type of vertebral fracture and the localisation, many novel instruments and surgical approaches are available to prevent complications such as vertebral malunion and neurological deficiencies through paraplegia [6].

Eventually, the surgical stabilization of an unstable vertebral fracture should provide the geriatric patient a preferably high level of quality of life-even ideally restore the status prior to injury. Up to now, only the consequences of morbidity and mortality following osteoporotic vertebral fractures were investigated in some studies, showing a reduced estimated survival 5 years after diagnosis (61\% compared with an expected value of 76\%) [7] and a subsequent reduction in activities of daily living with restriction of social contacts [8-10].

No valid data have been available yet with regard to the quality of life following the surgical treatment of traumatic unstable spinal fractures in geriatric patients. Thus, we decided to conduct this retrospective study in a proprietary patient population.

\section{Material and Methods}

The inclusion criteria for this study (evidence level 4) were an age of at least 65 years and a traumatic vertebral fracture type A3, B or C according to Magerl et al. [3] of the thoracic or lumbar spine, which had been surgically treated between 01/01/2004 and 31/12/2008 (5-year period). All diagnoses were preoperatively confirmed by conventional $\mathrm{X}$-ray images and CT scans. The exclusion criteria were fractures due to osteoporosis without preceding trauma and injuries of the cervical spine (which require specific consideration), age of less than 65 years at the time of surgery, and follow-up periods of less than 12 months post surgery. Based on these inclusion and exclusion criteria, the patient population was then identified in an electronic database of our department and initially analysed with regard to empirical data, including complications and neurological status according to Frankel et al. [11]. Then, the patients were contacted via telephone and, after giving their consent, they were invited for a clinical and radiological follow-

*Corresponding author: Müller Franz, Department of Trauma and Orthopaedic Surgery, University Medical Center, Germany, Tel: 0049-941-944-6805; Fax: 0049-941-944-6806; E-mail: muellerfj5@aol.com

Received July 02, 2012; Accepted August 05, 2012; Published August 10, 2012 Citation: Müller F, Füchtmeier B, Kinner B, Hierl J, Nerlich M, et. al. (2012) Health Related Quality of Life Outcome after Operative Treatment of Traumatic Vertebral Fractures in Geriatric Patients. J Trauma Treat 1:143, doi:10.4172/2167. 1222.1000143

Copyright: (C) 2012 Müller F, et al. This is an open-access article distributed unde the terms of the Creative Commons Attribution License, which permits unrestricted use, distribution, and reproduction in any medium, provided the original author and source are credited. 
up. Conventional radiological images in 2 planes for the analysis of the affected spinal section were made and a clinical follow-up investigating subjective satisfaction, Visual Analogue Scale scores for back and leg pain, homecare nursing, level of care, and SF-12 questionnaire results was conducted. The SF-12 questionnaire is a short form of the SF-36 questionnaire, which is well accepted for the evaluation of the general health-related quality of life and which has sufficient reliability and validity even in the German version $[12,13]$. The SF-12 contains 12 questions and their scores add up to a total between 0 and 100 for each of a physical and a mental summary measure. A high score represents a high quality of life; a lower score represents an equivalently lower quality of life. A score of 50 with a standard deviation of 10 is equivalent to the health status of the average population.

\section{Statistics}

SPSS version 18.0 for Windows (SPSS Inc, USA) was used for the statistical data analysis. The continuous variables, specified as average values with their standard deviations, were evaluated using the Shapiro-Wilk test for normal distribution. If $\mathrm{p}>0.05$ was associated with normal distribution, the t-test was used for comparisons to calculate significance if 2 samples were independent, and the t-test for paired samples was used if 2 samples were related. If $p$ was $<0.05$ in the Shapiro-Wilk test and no normal distribution was found, the Mann-Whitney-U test was used if the samples were independent, and the Wilcoxon test was used if 2 samples were related. The significance level was set at $<0.05$.

Pearson's correlation coefficient was used for the correlation calculation of samples with normal distribution and Spearman's Rho was used for ordinal scale samples with normal distribution. A correlation coefficient of $r<0.2$ is considered to represent a very low correlation, while $\mathrm{r}=0.2-0.5$ is considered to represent a low correlation. $\mathrm{r}>0.7$ represents a high correlation.

\section{Results}

A total of 42 patients (26 male, 16 female) underwent surgical treatment. The average age was $71.4 \pm 5.7$ years (range 65 to 87 years). Altogether, 31 out of 42 patients (74\%) gave their approval to followup, allowing us to conduct a complete analysis of the radiological and clinical outcome. However, 11 out of 42 patients (26\%) were not directly available for the following reasons: 3 gave no agreement for ambulant follow up, 3 were immobile and 5 patients had already died (with a mean survival time of $4.0 \pm 1.0$ months). Of these 11 patients, the following parameters were investigated via telephone, either by talking to the patients or to their relatives (deceased patients): satisfaction, homecare nursing, level of care, and survival time.

The classification showed mainly type A3 burst fractures (26 patients, $62 \%$ ), followed by type $B$ (11 patients) and type C (5 patients) injuries due to Magerl et al. [3].

The predominant localisation of the fracture was the level between T 11 and L 2 (28 patients, 66\%). The most common causes for injuries were falls from height ( 21 patients, $50 \%$ ), followed by minor traumas such as falling to the ground ( 15 patients, $36 \%$ ). No one had polytrauma, but 4 patients had multiple injuries.

Altogether, 35 patients received open bi-segmental, and 7 patients multi-segmental ( $>2$ segments) dorsal instrumentation by internal fixator, with additional spondylodesis using autologous spongiosa. Surgical treatment was performed within 48 to 72 hours post-trauma. An additional decompression by (hemi-) laminectomy was performed in cases of manifest, dislocated epidural bone fragment and neurological symptoms (14 out of 42 patients). An additional ventral stabilisation of the thoracolumbar spine (vertebral body replacement) was performed in 9 out of 42 patients during a second surgical procedure.

Surgical revision rate was $12 \%$ (5 patients) with the following complications: deep wound infection (3x), 1 impaired wound healing, and 1 pedicle screw displacement that required revision.

From a neurological point of view, 41 out of 42 patients were able to walk according to the classification by Frankel et al. [11] both prior to and post surgery; there was only one patient with trauma-related paraplegia, which did not improve post surgery.

The local Cobb angle [14]-radiologically measured on lateral X-ray images-improved from $-4.7^{\circ} \pm 12.3$ kyphosis prior to surgery to $-1.5^{\circ}$ \pm 11.1 post surgery. However, this was statistically not significant ( $\mathrm{p}$ $=0.107$; t test). In contrast, the postoperative loss of correction at the time of the follow-up worsened significantly from $-1.5^{\circ} \pm 11.1$ to $-8.5^{\circ}$ \pm 11.3 kyphosis $(\mathrm{p}<0.001$; $\mathrm{t}$ test $)$ (Figure 1$)$.

\section{Health-related quality of life}

Subjective patient satisfaction was evaluated clinically in 31 out of 42 patients (74\%) and via telephone in 11 out of 42 patients (26\%) using the 5-part question of the SSE Spine Tango [15] . On behalf of the deceased patients, the question was answered by their relatives: From a subjective point of view, 35 out of 42 patients (83\%) were satisfied or very satisfied with the treatment. The following reasons were given for dissatisfaction: Revision surgery and chronic pain (2x); symptoms of paraplegia, impaired mobility, and early postoperative death (1x each).

The patients' score (arithmetic average) for back pain on the Visual Analogue Scale with 11 sections (no pain=0; most severe pain=10) [15] was $3.2 \pm 2.7$. The arithmetic average for leg and gluteal pain on the Visual Analogue Scale was $2.0 \pm 2.5$.

A total of 28 out of 42 patients (67\%) experienced no change in their homecare nursing following surgery. However, 14 out of 42 patients (33\%), who had lived independently in their home prior to surgery, were depending on help from their relatives at the time of the follow-up. On the other hand, none of the patients were living in a nursing home at the time of the follow-up. From a statistical point of view, homecare nursing at the time of the follow-up was significantly worse when compared to the situation prior to trauma ( $p>0.001$; sign test)

The assignment to a care level (Level 0: No need for nursing, Level 1: Considerable need for nursing, Level 2: Need for a high level of

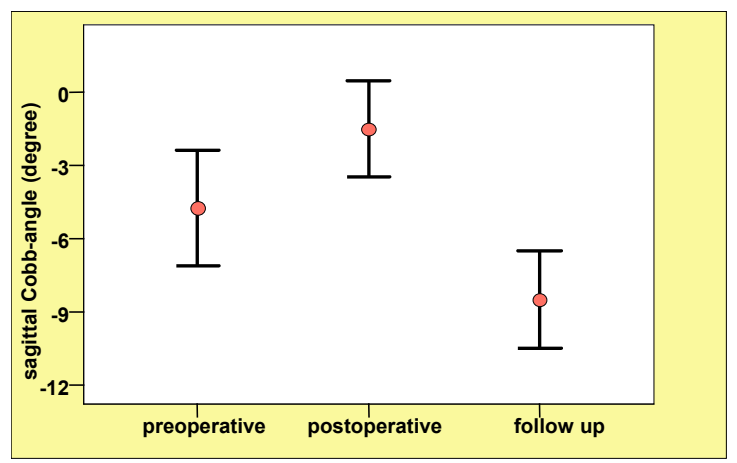

Figure 1: Local cobb angle on lateral X-ray images: Significant loss of correction was observed during the postoperative course. 
nursing, Level 3: Need for the highest level of nursing) at the time of the follow-up also worsened significantly compared to the situation prior to trauma ( $\mathrm{p}=0.008$; sign test). The assignment to a care level worsened post surgery in 8 out of 42 patients (19\%): 4 out of 42 patients and another 4 out of 42 patients had been assigned to a care level that was one or two levels higher than before. Another 7 out of 42 patients (16.7\%), who had not been assigned to a care level, had to be assigned to a care level post surgery. However, none of the patients was assigned to care level 3 at the time of the follow-up. The assignment to a care level at the time of the follow-up was significantly worse than prior to trauma ( $\mathrm{p}<0.001$, sign test).

Quality of life was evaluated using the SF-12 questionnaire, and the physical and mental summary measures were calculated for 31 out of 42 patients (74\%). The average age in the study's patient population was $73.8 \pm 5.4$ years (rang 66-89). Thus, the summary measure for 65 to 74 year-old persons without spinal disorders was used as a reference population for comparison [16]

The 31 patients showed a total score for physical health of $35.9 \pm$ 11.0 and a total score for mental health of $48.7 \pm 9.5$. The reference values [16] of 65-74 year-old persons for physical health and for mental health were $43.9 \pm 9.3$ and $51.6 \pm 8.4$, respectively.

Hence, the physical quality of life in the patient population after 27.3 months post surgery was significantly worse $(p<0.001)$ than in the general reference population. There was no significant difference between the patient population and the healthy reference population of 65-74 year-old persons $(\mathrm{p}=0.099)$.

The results of the physical and mental summary measures of the SF12 were evaluated with regard to their correlation with the postoperative loss of correction (as measured with the local Cobb angle [14] in lateral $\mathrm{X}$-ray images). However, no correlation $(\mathrm{r}<0.4$; Pearson or Spearman's Rho) between the physical and mental summary measures and the postoperative radiological loss of correction was found.

\section{Discussion}

The retrospective study with evidence level 4 was primarily conducted to assess the quality of life after the surgical stabilisation of unstable vertebral fractures in geriatric patients. The secondary objective was the assessment of postoperative morbidity and mortality.

Against this background, it was also necessary to include the surgical procedures and implants in the empirical data collection and to perform a radiological follow-up.

It must be emphasised here that, in the meantime, a wide range of different surgical approaches, procedures, and implants have become available for the treatment of traumatic vertebral fractures, including augmentation procedures and percutaneous methods. However, dorsal stabilisation using an internal fixator (with dorsal decompression, if required) is still considered the standard procedure. In our study population, this method was mostly used alone, although there were some patients who had also undergone vertebral body replacement. Nevertheless, a more detailed analysis of these methods was not a primary or secondary study objective.

We deliberately excluded all osteoporotic fractures without preceding trauma, since these injuries mainly represent stable type A1 fractures according to Magerl et al. [3]. An age of less than 65 years was another exclusion criterion. The main causes of injuries were falling from a height, followed by falling down stairs. It must be noted here that our study population included a high percentage of male patients.
So far, studies that targeted the investigation of functional outcome and quality of life of old patients, commonly concentrated on osteoporosisrelated vertebral fractures involving no or only minor instabilities and thus included mainly female patients in their study populations $[4,17]$.

The most common complications of spinal surgery are wound infections, implant failures, and pedicle screw misplacements, with the latter being related with the risk of iatrogenic damage of the spinal cord [17].

In our study, we identified 5 out of 42 patients (12\%) with complications requiring revision. One of these complications was a pedicle screw displacement requiring revision. No case of implant failure (e.g. pedicle screw loosening requiring revision) was documented during the period until the follow-up. Generally, the surgical revision rate of $12 \%$ in geriatric patients rather presents a low percentage [18].

Several studies, although with younger patient populations, have consistently shown that there is no correlation between the radiological loss of correction on the one side and clinical symptoms (mainly postoperative pain) on the other [19-21]. This also applies to our population for which the results of the SF-12 questionnaire were compared with the radiological loss of correction and analysed statistically without showing any correlation.

A multicentre study [22] investigated the patients' postoperative satisfaction using an ordinal scale with 4 categories: $31 \%$ of the patients were very satisfied, $52 \%$ were satisfied, $13 \%$ less satisfied, and $5 \%$ not satisfied.

At the time of the follow-up, the level of satisfaction in our patient population was also investigated using an ordinal scale with 5 levels. In total, $83 \%$ of the patients were satisfied. The percentage of unsatisfied patients was similar to that of the study mentioned before [22]: 17\%.

Particularly in elderly patients, the outcome of a surgical procedure should be evaluated based on postoperative quality of life, whereby the term quality of life describes a multidimensional construction comprising at least 4 components: mental health, physical health, social relations, and functional abilities [23,24]. The SF-36 questionnaire is an established measurement tool for the assessment of the general health-related quality of life, and it has been investigated for validity and reliability in various studies $[16,25,26]$. In various studies, this questionnaire was used to assess degenerative changes of the spine $[27,28]$, and it has been used in numerous studies with fractures of the thoracolumbar spine $[20,29,30]$. Thus, it can be used as a measurement tool for the quality of life in patients with spinal injuries.

The SF-12 questionnaire that we used is a short form of the SF-36 with a total of 12 questions that directly refer to physical and mental health $[25,26]$. Similar to the SF-36, a score of 0-100 can be achieved for each of the two dimensions (calculated by computer-assisted analysis software), in which a high score represents a high quality of life.

The patients of this study achieved a total score of $35.8 \pm 11.3$ for physical health and a total score of $49.3 \pm 10.0$ for mental health. The study population achieved significantly lower summary measures with regard to physical health when compared to a (American) reference population [16]. It is not yet clear to what extent the values of the American reference population match with a German reference population, since there are no valid data available yet. The summary measures with regard to mental health were not significantly different when compared with the reference population.

Fisher et al. [31] used the SF-36 questionnaire for the evaluation of 
Citation: Müller F, Füchtmeier B, Kinner B, Hierl J, Nerlich M, et. al. (2012) Health Related Quality of Life Outcome after Operative Treatment of Traumatic Vertebral Fractures in Geriatric Patients. J Trauma Treat 1:143. doi:10.4172/2167-1222.1000143

quality of life after surgery on the upper thoracic spine in 27 patients at an average age of 39.9 years and with an average follow-up of more than 3 years: In this study, the physical summary measurement was as low as 35.9 and the mental summary measurement was 56.4. Briem et al. [20] investigated 30 patients (range 18-63 years) with traumatic fractures of the thoracolumbar spine. Two years post surgery after dorso-ventral instrumentation the SF-36 results showed a physical score of $45.8 \pm 8.9$ and a mental score of $51.4 \pm 9.2$.

Cockerill et al. [32] used amongst others the SF-12 to investigate the quality of life of only 73 patients recruited from 12 European centers with an incident vertebral fracture. In this study a vertebral fracture was associated with impairment in quality of life compared with their matched controls. The average scores of 39.9 and 47.2, respectively, were achieved for physical and mental health. The average age (64.8 years) in this study was almost 10 years lower compared to our study population, which may explain the better result for physical health, while the result for mental health was similar.

Recently, Yaray et al. [33] also showed that the most healthy outcome scores (e.g. VAS, ODI, RMDQ) were significantly better in a healthy volunteers group compared to patients after surgical treatment of thoracolumbar fractures. Surprisingly, statistical significance has neither been shown in the SF-12 nor in the SF-36 questionnaire. Other authors [34,35] investigated the effects of various fracture localisations on quality of life. These studies showed that osteoporotic fractures of the spine or hip have more negative effects on quality of life than fractures of the humerus or wrist, and that patients with vertebral fractures have worse long-term results in the SF-36 questionnaire compared to patients with hip fractures.

The majority of our patients had no need for nursing (as defined by German social laws), neither before the index event nor at the time of the follow-up. On the other hand, the need for nursing was increased in 8 patients (19\%). As a consequence, these patients required support by family members or carers for their personal hygiene, nutrition, and mobility, with an expenditure of time between $1.5 \mathrm{~h}$ (care level 1) up to $3 \mathrm{~h}$ (care level 2). No patient required the maximum level of nursing (care level 3) 24 hours a day.

All in all, it can be stated that vertebral fractures generally reduce the physical quality of life of both young and elderly patients permanently-irrespective of the aetiology, severity of the injury, and treatment options.

Objective parameters, such as level of care and homecare nursing, were also taken into account in our study. Both of these parameters worsened significantly post surgery. In the literature, there are only studies [19,29-35] that evaluated the quality of life using a questionnaire, while not considering level of care and homecare nursing. Studies with younger patients who had undergone spinal surgery partially evaluated the outcome of the procedure based on the postoperative working life of the patients [17]. All patients of our study population had already retired prior to surgery. As a critical aspect, it must be emphasised that an age-related upgrading of the level of care and an increased need for homecare nursing may develop in elderly persons at an average age of more than 70 years-similar to our population-even without vertebral fracture, within a period of 2 years.

\section{Conclusions}

In our retrospective study, 42 geriatric patients at an average age of 71.4 years with unstable thoracolumbar vertebral fractures due to traumatic injuries were investigated. The patients received surgical treatment, mostly by dorsal internal fixator. The primary objective of the follow-up was the assessment of quality of life. After a mean period of 27.3 months post surgery, the need for nursing had significantly increased, and the quality of life as measured with the SF12 questionnaire was significantly reduced when compared to a healthy reference population. Thus, traumatic injuries of the spine in geriatric patients represent events that lead to a significantly increased need for nursing and in the medium term to a reduced quality of life when compared to an age-matching general population.

\section{References}

1. Lane NE (2006) Epidemiology, etiology, and diagnosis of osteoporosis. Am J Obstet Gynecol 194: S3-S11.

2. Grazio S (2006) Epidemiology of osteoporosis. Reumatizam 53: 18-31.

3. Magerl F, Aebi M, Gertzbein SD, Harms J, Nazarian S (1994) A comprehensive classification of thoracic and lumbar injuries. Eur Spine J 3: 184-201.

4. Ploeg WT, Veldhuizen AG, The B, Sietsma MS (2006) Percutaneous vertebroplasty as a treatment for osteoporotic vertebral compression fractures: a systematic review. Euro Spine J 15: 1749-1758.

5. Klezl Z, Clamp JA, Becker J, Jones M, Calthorpe D, et al. (2011) Impact of kyphoplasty treatment for vertebral compression fractures on pain and function in 105 patients. Acta Chir Orthop Traumatol Cech 78: 551-555.

6. Ponnusamy KE, lyer S, Gupta G, Khanna AJ (2011) Instrumentation of the osteoporotic spine: biomechanical and clinical considerations. Spine J 11: 54 63.

7. Cooper C, Atkinson EJ, Jacobsen SJ, O`Fallon WM, Melton LJ 3rd (1993) Population-based study of survival after osteoporotic fractures. Am J Epidemio 137: 1001-1005.

8. Gold DT (1996) The clinical impact of vertebral fractures: Quality of life in woman with osteoporosis. Bone 18: 185S-189S

9. Center JR, Nguyen TV, Schneider D, Sambrook PN, Eisman JA (1999) Mortality after all major types of osteoporotic fracture in men and women: an observational study. Lancet 353: 878-882.

10. Pluijm SM, Tromp AM, Smit JH, Deeg DJ, Lips P (2000) Consequences of vertebral deformities in older men and women. J Bone Miner Res 15: 1564 1572.

11. Frankel HL, Hancock DO, Hyslop G, Melzak J, Michaelis LS, et al. (1969) The value of postural reduction in the initial management of closed injuries of the spine with paraplegia and tetraplegia. I. Paraplegia 7: 179-192.

12. Gandek B, Ware JE, Aaronson NK, Apolone G, Bjorner JB, et al. (1998) Cross-validation of item selection and scoring for the SF-12 Health Survey in nine countries: results from the IQOLA Project. International Quality of Life Assessment. J Clin Epidemiol 51: 1171-1178.

13. Bullinger M (1995) German translation and psychometric testing of the SF-36 Health Survey: preliminary results from the IQOLA Project. International quality of life assessment. Soc Sci Med 41: 1359-1366.

14. Cobb IR (1948) Outline for the studying of scoliosis. Instr Cours Lect 5: 261 275.

15. Röder C, Chavanne A, Mannion AF, Grob D, Aebi M (2005) SSE Spine Tangocontent, workflow, set-up. www.eurospine.org -Spine Tango. Eur Spine J 14 920-924.

16. Ware JE, Kosinski M, Turner-Bowker DM, Gandek B (2002) How to Score Version 2 of the SF-12 Health Survey (with a supplement documenting version 1). Lincoln, RI: QualityMetric Inc, Boston.

17. Rompe JD, Eysel P, Hopf C, Heine J (1995) Transpedicular lumbar spondylodesis in elderly patients. Z Orthop Ihre Grenzgeb 133: 249-255.

18. Konstantinidis L, Mayer E, Strohm PC, Hirschmüller A, Südkamp NP, et al (2010) Early surgery-related complications after anteroposterior stabilization of vertebral body fractures in the thoracolumbar region. J Orthop Sci 15: 178-184.

19. Knop C, Blauth M, Bastian L, Lange U, Kesting J, et al. (1997) Fractures of the thoracolumbar spine. Late results of dorsal instrumentation and its consequences. Unfallchirurg 100: 630-639.

20. Briem D, Linhart W, Lehmann W, Bullinger M, Schoder V, et al. (2003) 
Citation: Müller F, Füchtmeier B, Kinner B, Hierl J, Nerlich M, et. al. (2012) Health Related Quality of Life Outcome after Operative Treatment of Traumatic Vertebral Fractures in Geriatric Patients. J Trauma Treat 1:143. doi:10.4172/2167-1222.1000143

Investigation of the health-related quality of life after a dorso ventral stabilization of the thoracolumbar junction. Unfallchirurg 106: 625-632.

21. Katscher S, Verheyden P, Gonschorek O, Glasmacher S, Josten C (2003) [Thoracolumbar spine fractures after conservative and surgical treatment Dependence of correction loss on fracture level]. Unfallchirurg, 106: 20-27.

22. Knop C, Blauth M, Bühren V, Arand M, Egbers HJ, et al. (2001) Surgica treatment of injuries of the thoracolumbar transition - 3: Follow-up examination. Results of a prospective multi-center study by the "Spinal" Study Group of the German Society of Trauma Surgery. Unfallchirurg 104: 583-600.

23. Bullinger M (1996) [Assessment of health related quality of life with the SF-36 Health Survey]. Rehabilitation (Stuttg) 35: 17-27.

24. Bombardier C (2000) Outcome assessments in the evaluation of treatment of spinal disorders: summary and general recommendations. Spine 25: 31003103.

25. Ware JE, Kosinski M, Keller SD (1995) SF-12: How to Score the SF-12 Physical and Mental Health Summary Scales. (2ndedn), Boston, MA: The Health Institute, New England Medical Center.

26. Ware J Jr, Kosinski M, Keller SD (1996) A 12-Item Short-Form Health Survey: Construction of scales and preliminary tests of reliability and validity. Med Care 34: $220-233$

27. Nickel R, Egle UT, Eysel P, Rompe JD, Zöllner J, et al. (2001) Health-related quality of life and somatization in patients with long-term low back pain: a prospective study with 109 patients. Spine 26: 2271-2277.
28. Sjölie AN (2002) Psychosocial correlates of low-back pain in adolescents. Eur Spine J 11: 582-588.

29. Fisher CG, Noonan VK, Smith DE, Wing PC, Dvorak MF (2005) Motor recovery functional status, and health-related quality of life in patients with complete spinal cord injuries. Spine 30: 2200-2207.

30. Dvorak MF, Fisher CG, Aarabi B, Harris MB, Hurbert RJ, et al. (2007) Clinica outcomes of 90 isolated unilateral facet fractures, subluxations, and dislocations treated surgically and nonoperatively. Spine 32: 3007-3013.

31. Fisher C, Singh S, Boyd M, Kingwell S, Kwon B, et al. (2009) Clinical and radiographic outcomes of pedicle screw fixation for upper thoracic spine (T15) fractures: a retrospective cohort study of 27 cases. J Neurosurg Spine 10 207-213.

32. Cockerill W, Lunt M, Silman AJ, Cooper C, Lips P, et al. (2004) Health-related qualitiy of life and radiographic vertebral fracture. Osteoporos Int 15: 113-119.

33. Yaray O, Akesen B, Aydinli U (2011) Long-term outcome after surgical treatment of thoracolumbar fractures versus a control group of healthy volunteers. Acta Orthop Belg 77: 93-96.

34. Hallberg I, Rosenqvist AM, Kartous L, Löfman O, Wahlström O, et al. (2004) Health-related quality of life after osteoporotic fractures. Osteoporos Int 15 834-841.

35. Adachi JD, Adami S, Gehlbach S, Anderson FA Jr, Boonen S, et al. (2010) Impact of prevalent fractures on quality of life: baseline results from the global longitudinal study of osteoporosis in woman. Mayo Clin Proc 85: 806-813. 